\title{
A Rectangular Cuboid Satellite Module Layouts Method Based on Integer Optimization
}

\author{
Junjie Yang ${ }^{1,}$, , Xiaoqian Chen ${ }^{2, b}$ and Wen Yao ${ }^{3, c}$ \\ ${ }^{1,2,3}$ College of Aerospace Science and Engineering, National University of Defense Technology, \\ Changsha, China

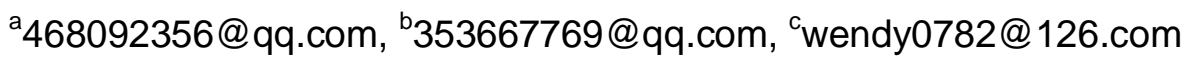

\begin{abstract}
Keywords: satellite; layout optimization; integer optimization
Abstract. According to the module layout design properties problem of rectangular cuboid satellite, a new layout optimization strategy of satellite module with constraints is proposed. Firstly, taking the module layout design of rectangular cuboid satellite as background, the mathematical model of satellite module layout optimization is constructed. Then the layout order of packing modules is regarded as the design variable with introducing the idea of integer optimization. Finally, this layout optimization strategy is applied to an example of satellite module layout design and simulation results demonstrate the efficacy and efficiency of this layout optimization strategy. This strategy can effectively reduce the difficulty of satellite module layout design and provide much to improve the efficiency of rectangular cuboid satellite overall design .
\end{abstract}

\section{Introduction}

The satellite module layout problem (SMLD) contains several mutual-conflicting objectives and constraints. It is known as its engineering complexity and combinational explosion of computational complexity and belongs to the constrained multi-objective optimization problem (MOP) [1]. It is almost impossible to be fully solved only by engineers' experience and intuition and little literature is available on this topic.

Over the past decades, a number of evolutionary algorithms have been suggested to solve the SMLD. Kamaran and Maziar [2] proposed a FARAGAM algorithm for the arrangement of functional subassemblies of the satellite ZS3-SAT. Teng [3, 4] proposed a set of heuristics with the support of sensitivity analysis technique for a packing problem with dynamical equilibrium constraints. Jackson and Norgard [5] introduced two important issues to be taken into consideration in the module layout problem: the balance of module heat dissipation over the satellite panels and the electrical interconnection between individual devices, etc. The design variables of the methods listed above are locations of all module, and the dimension of the design variables is so high that results low computing efficiency. This paper presents a module layout optimization strategy for cuboid satellite based on integer optimization, which takes the installation sequence as the design variable and reduces the computing complexity largely.

\section{Problem statement}

Because of the space utilization of rectangular cuboid satellite is much higher than cylindrical (or conical) satellite, the object of study in this paper is the layout of module in the rectangular cuboid satellite, as shown in Fig 1, which is different from the rotating frustum vessel shown in the article of Teng [6].

The optimal layout problem of a simplified rectangular cuboid satellite, such as Tian-Tuo1 which is a one board satellite made by National University of Defense Technology, can be described as follows: A total number of $n$ objects which are simplified as rectangular cuboids and cylinders must be located within a rectangular cuboid satellite frame shown in Fig 1(a). The upper and lower surfaces of one bearing plate (whose area is not predefined) is used to fix all the objects shown in Fig 1(b). The conditions that should be satisfied in the course of layout and the optimization objectives are as follows: 
(a) The satellite frame and objects are regarded as rigid bodies. All of the objects should be contained within the satellite frame.

(b) No overlap exists among the objects.

(c) The center of mass of the system should be as near as possible to the center of the rectangular cuboid frame, so that some dynamical features are ensured.

(d) The enveloping area of the envelop rectangle (which is area of the bearing plate) should be as small as possible to improve the space utilization and reduce the manufacturing cost of the satellite.

(e) The smallest height of the satellite shell should be as small as possible.

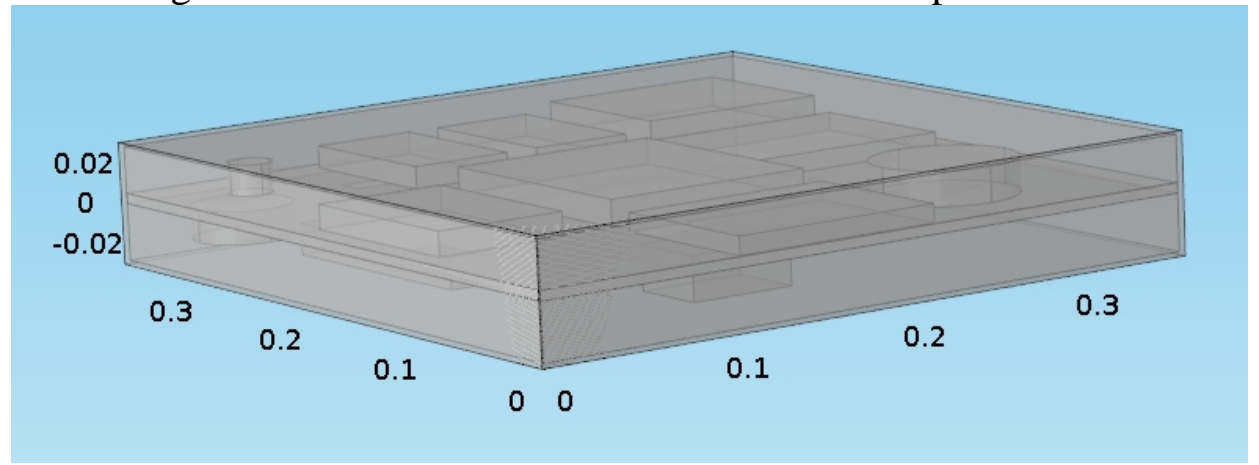

(a)

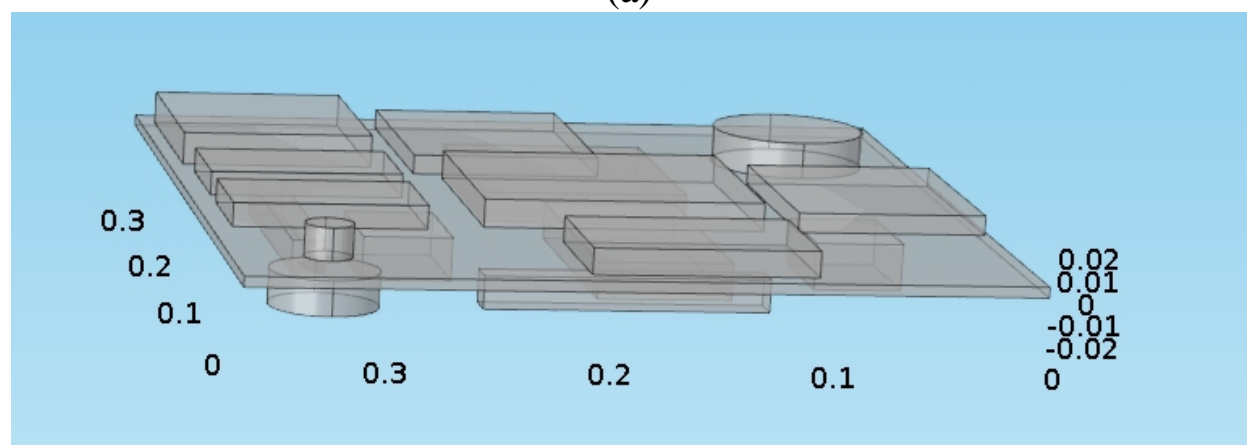

(b)

Fig 1. (a) The schematic diagram of the satellite frame;

(b) The schematic diagram of locating objects within a rectangular cuboid satellite.

As an optimization problem, the design variable $S=\left\{q_{1}, q_{2}, q_{3} \ldots q_{n}\right\}$ is the sequence of the items to be packed, for example $q_{i}(i=1,2,3 \ldots n)$ represents the serial number of the $i_{t h}$ packed object is $q_{i}$. The constraints are (a), (b) and (c), while the optimization objectives are (d) and (e). An arrangement is said to be feasible when no items overlap and all items are placed completely within the container and parallel to the container edges, and considering the geometry features of rectangle cuboid and cylinder, the cylindrical objects are all represented by rectangular cuboids whose lengths are equal to widths.

\section{Optimization strategy}

The optimization strategy presented in this paper concludes two levels: one is the distribution of the objective items which part should be located on the up base of the bearing plate and which part should be located on the down base; the second level is to allocate the items of up base and down base.

Distribution of objects between bearing surfaces. The first level is to distribute which part of objects to be packed on the up base and the others to be packed on the down base. Deonte the mass, length, width and height of object whose serial number is $q_{i}$ as $m_{q_{i}}, l_{q_{i}}, w_{q_{i}}$ and $h_{q_{i}}$ respectively.

Calculate Sum_mh1 in Eq. (1) until the Sum_mhl close to the half of Sum_mh for a given sequence $S=\left\{q_{1}, q_{2}, q_{3} \ldots q_{n}\right\}$ and record $t$. And this step is used to ensure the mass center of the $\mathrm{Z}$-axis, denoted as $z_{c}$, close to the shape center of the satellite frame which is denoted as $z_{s}$. 
Sum_mh1 $=\sum_{i=1}^{t} m_{q_{i}} \times h_{q_{i}} ;$ Sum_ $m h=\sum_{i=1}^{n} m_{q_{i}} \times h_{q_{i}}$.

Then cut the sequence $S=\left\{q_{1}, q_{2}, q_{3} \ldots q_{n}\right\}$ into two sequence $S_{-} u p=\left\{q_{1}, q_{2}, q_{3} \ldots q_{t}\right\}$ and $S_{-}$down $=\left\{q_{t+1}, q_{t+2}, q_{t+3} \ldots q_{n}\right\}$. Denote the largest height of the objects to be packed in $S_{-}$up and $S_{-}$down as $h_{\text {max }_{\imath} u p}$ and $h_{\text {max_down }}$ respectively.

And the smallest height of the satellite shell mentioned above can be written as Eq. (2) to ensure the mass center of $\mathrm{z}$-axis is equal to the shape center of $\mathrm{z}$-axis.

$$
H=2 \times \max \left(h_{\text {max }_{-} u p}+\text { Sum_mh-2Sum_mh1, } h_{\text {max }_{-} d o w n}-S u m_{-} m h+2 S u m \_m h 1\right) .
$$

Detailed layout design within surfaces. In the second level, the MOGA\& SBFFA algorithm is used to transform the one $3 \mathrm{D}$ problem to two $2 \mathrm{D}$ problems.

MOGA is the abbreviation of multiple objective genetic algorithm. SBFFA is the abbreviation of self-boundary fall free algorithm which is shown in Fig 2. The schematic graph of SBFFA shows the compactness and non-interference of the layout. Especially, the non-interference of this algorithm reduces a lot computational expense which has been compared in [7]. The interference volumes among objects and between objects and satellite frame, denoted as $V$, are 0 by using

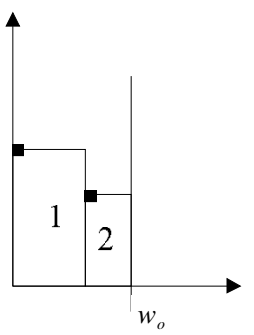

(a)

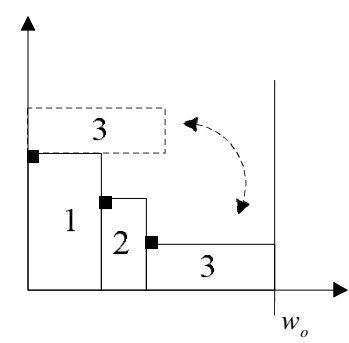

(b)

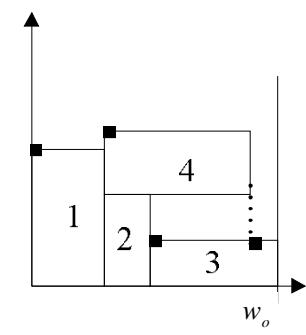

(c)

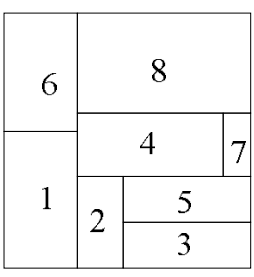

(d)

SBFFA.

Fig 2. The schematic graph of SBFFA.

After this step, the layouts of modules of the up base and down base are basically completed. Record the max length, denoted as $l_{\max }$, and max width, denoted as $w_{\max }$, of the enveloping rectangles of the up base and down base. The area of enveloping rectangle (the area of bearing plate), denoted as $A$, can be calculated by Eq. (3).

$A=l_{\text {max }} * w_{\text {max }}$

Calculate the mass center of up base and down base respectively. The modules on one base whose area is smaller should move to balance the mass center of the whole satellite until the mass center is equal to the shape center. Denote the mass center of the whole satellite is $\left(x_{c}, y_{c}, z_{c}\right)$, while the shape center of the satellite is $\left(x_{s}, y_{s}, z_{s}\right)$. The distance between mass center and shape center is denoted as $D$. And this step is to ensure the mass center coincides the shape center.

$$
D=\sqrt{\left(x_{c}-x_{s}\right)^{2}+\left(y_{c}-y_{s}\right)^{2}+\left(z_{c}-z_{s}\right)^{2}}=0
$$

The optimization problem can be formulated as follows:

Design variable: $S=\left\{q_{1}, q_{2}, q_{3} \ldots q_{n}\right\}$

$\min : A, H$

s.t. $\quad D=0, V=0$

In the MOGA\&SBFFA algorithm, GA products the serial number of the items. Then the SBFFA places the items logically and MO strategy outputs the Pareto solution of the layout problem. And the flow chart is shown in Fig 3. 


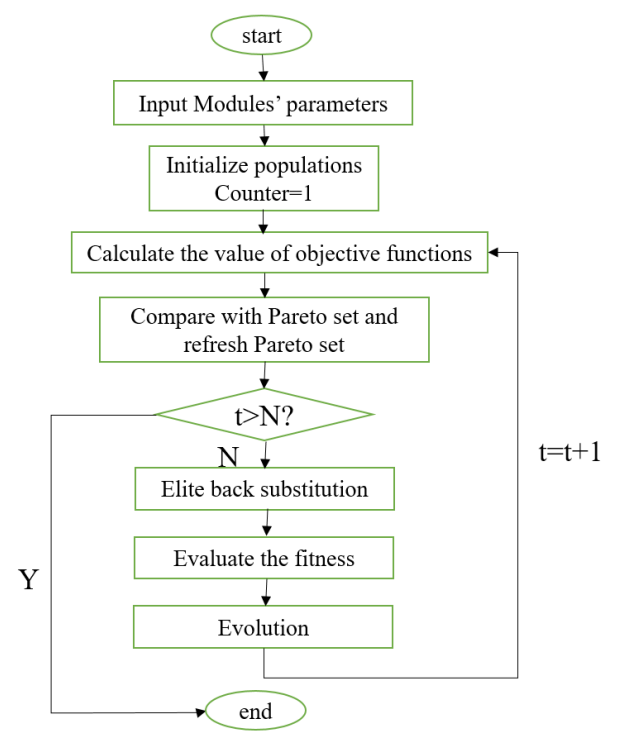

Fig 3. The flow chart of MOGA\&SBFFA

\section{Experiment result}

Based on the optimization algorithm presented above, Matlab codes have been written to simulate the layout of one board satellite as shown in Fig 1. The number of objective items is 26 and the parameters of the items is listed in Table 1[8]. In Table 1, M is mass, $\mathrm{L}$ is length, $\mathrm{W}$ is width and $\mathrm{H}$ is height. The unit of mass is $\mathrm{kg}$ and the unit for the other three is $\mathrm{mm}$.

Table 1 Mass and geometric parameters of objective items in satellite

\begin{tabular}{|l|l|l|l|l|l|l|l|l|l|l|l|l|l|l|}
\hline No. & M & L & W & H & No. & M & L & W & H & No. & M & L & W & H \\
\hline 1 & 4.0 & 730 & 730 & 50 & 10 & 1.2 & 250 & 150 & 32 & 19 & 2.6 & 360 & 360 & 43 \\
\hline 2 & 4.5 & 850 & 600 & 63 & 11 & 1.2 & 260 & 140 & 20 & 20 & 1.0 & 160 & 160 & 30 \\
\hline 3 & 3.6 & 500 & 450 & 76 & 12 & 1.1 & 200 & 160 & 21 & 21 & 1.5 & 130 & 130 & 34 \\
\hline 4 & 3.0 & 580 & 430 & 33 & 13 & 0.8 & 170 & 140 & 18 & 22 & 0.7 & 90 & 90 & 10 \\
\hline 5 & 3.9 & 760 & 560 & 64 & 14 & 4.0 & 450 & 450 & 64 & 23 & 2.0 & 600 & 600 & 40 \\
\hline 6 & 2.1 & 350 & 350 & 36 & 15 & 2.0 & 400 & 400 & 73 & 24 & 1.5 & 450 & 450 & 30 \\
\hline 7 & 3.3 & 700 & 270 & 45 & 16 & 3.2 & 250 & 250 & 34 & 25 & 1.8 & 500 & 450 & 40 \\
\hline 8 & 2.0 & 660 & 280 & 16 & 17 & 2.6 & 340 & 340 & 70 & 26 & 2.5 & 400 & 400 & 40 \\
\hline 9 & 1.8 & 500 & 350 & 56 & 18 & 1.4 & 150 & 150 & 50 & & & & & \\
\hline
\end{tabular}

Compared with the other 6 algorithm in article [8], the best performance indexes of satellite layout scheme is listed below, as shown in Table 2.

Table 2. Results of seven algorithms for satellite layout

\begin{tabular}{|c|c|c|c|c|c|}
\hline Algorithms & $\begin{array}{c}\text { Interference } \\
\text { Volume }\left(\mathrm{mm}^{3}\right)\end{array}$ & $\begin{array}{c}\text { Unbalanced } \\
\text { Force(N) }\end{array}$ & $\begin{array}{c}\text { Enveloping } \\
\text { Area }\left(\mathrm{mm}^{2}\right)\end{array}$ & $\begin{array}{c}\text { Smallest } \\
\text { Height(mm) }\end{array}$ & $\begin{array}{c}\text { Computation } \\
\text { Time(s) }\end{array}$ \\
\hline Traditional GA & 0 & 0.13 & $2437^{2}$ & $/$ & 262 \\
\hline PSO & 0 & 0 & $2361^{2}$ & $/$ & 270 \\
\hline CCGA & 0 & 0.04 & $2368^{2}$ & $/$ & 274 \\
\hline CEPSO & 0 & 0 & $2327^{2}$ & $/$ & 275 \\
\hline PPSO & 0 & 0 & $2267^{2}$ & $/$ & 280 \\
\hline CEPSO_HR & 0 & 0 & $2251^{2}$ & $/$ & 291 \\
\hline MOGA\&SBFFA & 0 & 0 & $1600 * 2000$ & 161.4 & 33 \\
\hline
\end{tabular}


The best layout diagram of MOGA\&SBFFA is shown in Fig 4, the blue rectangles with numbers are the items to be packed and the black bold rectangle is the enveloping rectangle which presents the bearing plate in satellite.
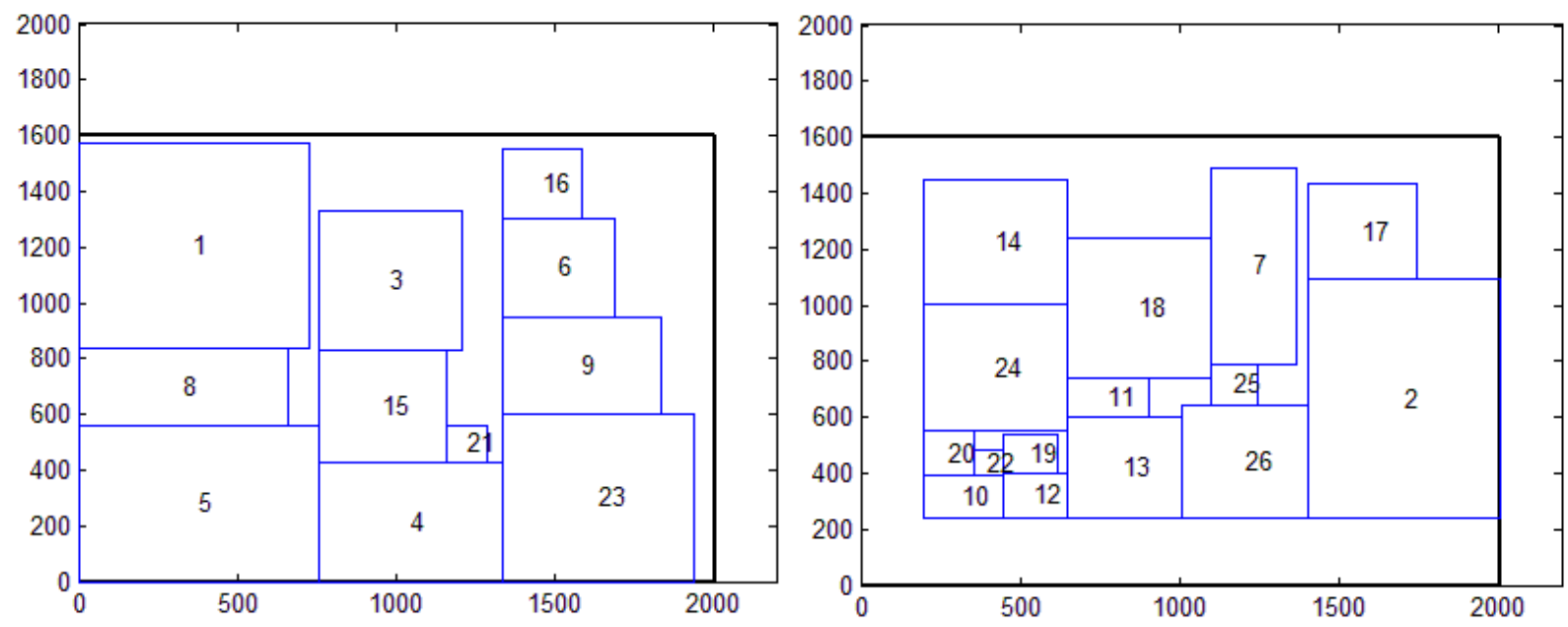

Fig 4. Best layout diagram of MOGA\&SBFFA for satellite layout

According to the results in Table 2, the performance of MOGA\&SBFFA is obvious. The computation time is almost $1 / 10$ of the other 6 methods' and the enveloping area of MOGA\&SBFFA is smallest meanwhile the other indexes are requirement-satisfied.

\section{Conclusion}

Considering the requirements of mass center, enveloping area and so on, MOGA\&SBFFA is proposed to solve the layout problem. Compared with the methods whose variables are position coordinates of the items, the method proposed in this paper use the allocation sequence of items as the design variable and reduces the computational expense largely. The results of the experiment demonstrate the correctness and high effectiveness of the proposed layout optimization strategy. This strategy can effectively release the difficulty of satellite module layout design and provide much to improve the efficiency of satellite overall design.

\section{Acknowledgements}

This work was financially supported by the National Natural Science Foundation of China (51205403).

\section{References}

[1] Ana, P. C., Fabiano, L. Optimization Engineering, 16: 165-181. (2015).

[2] Kamran, D., Maziar, A. and Hossein, S. F. FARAGAM algorithm in satellite layout. In: Proceeding of the 6th Asia-Pacific Conference on Multilateral Cooperation in Space Technology and Applications, Chinese Science Press, Beijing, China, pp. 120-127. (2001).

[3] Teng, H. F., Zhang, B., Shi, Y. J . Applied Soft Computing. 10(8):507-521. (2008).

[4] Teng, H. F., Sun, S. L., Liu, D. Q., et al. Computers and Operations Research, 28(6), 521-535. (2001).

[5] Jackson B, Norgard J (2002) A stochastic optimization for determining spacecraft avionics box placement. In: IEEE Aerospace Conference, vol. 5, pp 2373-2382. (2002).

[6] Teng, H.F., Chen, Y., Zeng, W. IEEE Transaction on Evolutionary Computation. 14(3):438-455. (2010). 
[7] Yang, J.J., Chen, X.Q., Wang, N., Qi, J. A self-boundary fall genetic algorithm for 2D open dimension rectangle packing problem of satellite layout design. In: Proceeding of the 6th International Conference on Astra-dynamics Tools and Techniques, Darmstadt, Germany. (2016).

[8] Zhang, B. Particle swarm optimization algorithm for satellite module layout optimization. Dalian University of Technology. (2006). 